\title{
Gauge Fixing in the Maxwell Like Gravitational Theory in Minkowski Spacetime and in the Equivalent Lorentzian Spacetime
}

\author{
Roldão da Rocha(1) and Waldyr A. Rodrigues Jr. ${ }^{(2)}$ \\ ${ }^{(1)}$ Centro de Matemática, Computação e Cognição \\ Universidade Federal do ABC, 09210-170, Santo André, SP, Brazil \\ roldao.rocha@ufabc.edu.br \\ ${ }^{(2)}$ Institute of Mathematics, Statistics and Scientific Computation \\ IMECC-UNICAMP CP 6065 \\ 13083-859 Campinas, SP, Brazil \\ walrod@ime.unicamp.br or walrod@mpc.com.br
}

December 122014

\begin{abstract}
In a previous paper we investigate a Lagrangian field theory for the gravitational field, which is there represented by a section $\left\{\mathfrak{g}^{\alpha}\right\}$ of the coframe bundle over Minkowski spacetime $\left(M \simeq \mathbb{R}^{4}, \stackrel{\circ}{\boldsymbol{g}}, \stackrel{\circ}{D}, \tau_{\circ}, \uparrow\right)$. Such theory, under appropriate conditions, has been proved to be equivalent to a Lorentzian spacetime structure $\left(M \simeq \mathbb{R}^{4}, \mathbf{g}, D, \tau_{\mathrm{g}}, \uparrow\right)$ where the metric tensor $\mathbf{g}$ satisfies the Einstein field equation. Here, we first recall that according to quantum field theory ideas gravitation is described by a Lagrangian theory of a possible massive graviton field (generated by matter fields and coupling also to itself) living in Minkowski spacetime. The massive graviton field is moreover supposed to be represented by a symmetric tensor field $\mathbf{h}$ carrying the representations of spin two and zero of the Lorentz group. Such a field, then (as it is well known) must necessarily satisfy the gauge condition given by Eq.(10) below. Next, we introduce an ansatz relating $\mathbf{h}$ with the 1 -form fields $\left\{\mathfrak{g}^{\alpha}\right\}$. Then, using the Clifford bundle formalism we derive from our Lagrangian theory the exact wave equation for the graviton and investigate the role of the gauge condition given by Eq.(10) by asking the question: does Eq.(10) fix any gauge condition for the field $g$ of the effective Lorentzian spacetime structure $\left(M \simeq \mathbb{R}^{4}, \mathbf{g}, D, \tau_{g}, \uparrow\right)$ that represents the field $\mathbf{h}$ in our theory? We show that no gauge condition is fixed a priory, as it is the case in General Relativity. Moreover we prove that if we use Logunov gauge condition, i.e., $\stackrel{\circ}{D}_{\gamma}\left(\sqrt{-\operatorname{det} g} g^{\gamma \kappa}\right)=0$ then the only a restricted class of coordinate systems (including harmonic ones) are allowed by the theory.
\end{abstract}




\section{Introduction}

In a previous pape1 1 , using the Clifford bundle formalism, a Lagrangian theory of the gravitational field as field in the Faraday sense, i.e., an object of the same ontology as the electromagnetic field living on a Minkowski spacetime structure $\mathbf{M}=\left(M \simeq \mathbb{R}^{4}, \stackrel{\circ}{g}, \stackrel{\circ}{D}, \tau_{\mathrm{g}}, \uparrow\right)$ has been formulated. The theory has been constructed on two assumptions. The first one is that the gravitational field is represented by a coframe $\left\{\mathfrak{g}^{\alpha}\right\}$, with $\mathfrak{g}^{\alpha} \in \sec \bigwedge^{1} T^{*} M \hookrightarrow \sec \mathcal{C} \ell(M, \stackrel{\mathrm{g}}{\mathrm{g}})$ whose dynamics is encoded in a Lagrangian density $\mathcal{L}_{g}$ (see Eq.(14) below) which is of the Yang-Mills type (containing moreover a gauge fixing term and an auto interaction term related to the "vorticity" of the fields). The theory is invariant under diffeomorphisms and under local Lorentz transformations of the coframe $\left\{\mathfrak{g}^{\alpha}\right\}$. The gravitational field couples universally with the matter fields and in such a way that the coupling resulting from the presence of energymomentum due to matter fields in some region of Minkowski spacetime distorts the Lorentz vacuum 3 in much the same way that stresses in an elastic body produces plastic deformations in it [17. To present some additional details we need to introduce some notation. So, let $\left\{\mathrm{x}^{\mu}\right\}$ be a set of global coordinates 4 for $M$ in the Einstein-Lorentz-Poincaré gauge associated to arbitrary inertial reference fram $I=\partial / \partial \mathrm{x}^{0} \in \sec T M$. Let $\left\{\partial / \partial \mathrm{x}^{\mu}\right\}$ be orthonormal basis for $T M$ and $\left\{\gamma^{\mu}=d \mathrm{x}^{\mu}\right\}$ the corresponding dual basis for $T^{*} M$. We take $\gamma^{\mu} \in \sec \bigwedge^{1} T^{*} M \hookrightarrow \sec \mathcal{C} \ell(M, \mathrm{~g})$. Of course, we have

$$
\stackrel{\circ}{g}=\eta_{\alpha \beta} \gamma^{a} \otimes \gamma^{\beta}, \quad \stackrel{\circ}{g}=\eta^{\alpha \beta} \frac{\partial}{\partial \mathrm{x}^{\alpha}} \otimes \frac{\partial}{\partial \mathrm{x}^{\beta}},
$$

and we recall that to each (non degenerated) metric tensor, say g $\in \sec T_{0}^{2} M$ there corresponds an unique invertible metric extensor field $\stackrel{\circ}{g}: \sec \bigwedge^{1} T^{*} M \rightarrow$ sec $\bigwedge^{1} T^{*} M$, while the metric tensor $\stackrel{\circ}{\mathrm{g}} \in \sec T_{2}^{0} M$ is represented by the extensor

\footnotetext{
${ }^{1}$ Please, consult the arXiv version of 7 which corrects an error of the printed version. See also 2 .

${ }^{2}$ Minkowski spacetime will be called Lorentz vacuum, in what follows. Moreover in the 5uple $\left(M \simeq \mathbb{R}^{4}, \stackrel{\circ}{\mathrm{g}}, \stackrel{\circ}{D}, \tau_{\mathrm{g}}, \uparrow\right), \stackrel{\circ}{\mathrm{g}}$ is a Minkowski metric, $\stackrel{\circ}{D}$ is its Levi-Civita connection, $\tau_{\mathrm{g}}$ is the volume element defining a global orientation and $\uparrow$ refers to a time orientation. The objects in the Lorentzian spacetime structure $\mathbf{L}=\left(M \simeq \mathbb{R}^{4}, \boldsymbol{g}, D, \tau_{\mathrm{g}}, \uparrow\right)$ have similar meanings. In what follows $\mathrm{g}$ denotes the metric of the cotangent bundle relative to the structure $\mathbf{M}$. If $\stackrel{\circ}{g}=\stackrel{\circ}{g}^{\kappa \iota} \partial_{\kappa} \otimes \partial_{\iota}$ and $\stackrel{\circ}{g}=\stackrel{\circ}{g}_{\kappa \iota} d x^{\kappa} \otimes d x^{\iota}$ then $\stackrel{\circ}{g}^{\kappa \iota} \stackrel{\circ}{g}_{\iota \xi}=\delta_{\xi}^{\kappa}$. Also g denotes the metric of the cotangent bundle relative to the structure $\mathbf{L}$ and if $\mathbf{g}=g^{\kappa \iota} \partial_{\kappa} \otimes \partial_{\iota}$ and $\boldsymbol{g}=g_{\kappa \iota} d x^{\kappa} \otimes d x^{\iota}$, then $g^{\kappa \iota} g_{\iota \xi}=\delta_{\xi}^{\kappa}$ More details, if needed are given, e.g., in [6].

${ }^{3}$ A region of Minkowski spacetime void of matter fields will be called Lorentz vacuum [2].

${ }^{4}$ If $\left\{\mathbf{x}^{\mu}\right\}$ and $\left\{\mathbf{x}^{\prime \mu}\right\}$ are global coordinate functions in the Einstein-Lorentz-Poincaré gauge, the coordinates of $\mathfrak{e} \in M$ in are $\left\{\mathrm{x}^{\mu}\right\}:=\left\{\mathrm{x}^{\mu}(\mathfrak{e})\right\},\left\{\mathrm{x}^{\prime \mu}\right\}:=\left\{\mathrm{x}^{\prime \mu}(\mathfrak{e})\right\}$ and $\mathrm{x}^{\prime \mu}=\Lambda_{\nu}^{\mu} \mathrm{x}^{\nu}$, with $\Lambda_{\nu}^{\mu}$ a proper and orthochronous Lorentz transformation.

${ }^{5}$ An inertial reference frame satisfies $\stackrel{\circ}{D} I=0$. See [6] for details.

${ }^{6} \wedge^{p} T^{*} M$ denotes the bundle of $p$-forms, $\wedge T^{*} M=\bigoplus_{p=0}^{4} \wedge^{p} T^{*} M$ is the bundle of multiform fields, $\mathcal{C} \ell(M, \stackrel{\mathrm{g}}{\mathrm{g}})$ denotes the Clifford bundle of differential forms. The symbol sec means section. All 'tricks of the trade' necessary for performing the calculations of the present paper are described in [6].
} 
field $\stackrel{\circ}{g}^{-1}: \sec \bigwedge^{1} T^{*} M \rightarrow \sec \bigwedge^{1} T^{*} M$. Our second assumption is that there is a plastic distortion field described by an extensor field $h: \sec \bigwedge^{1} T^{*} M \rightarrow$ sec $\bigwedge^{1} T^{*} M$ that distorts the cosmic lattice represented by the $\gamma^{a}$ producing the fields $\mathfrak{g}^{\alpha}$ such that

$$
\mathfrak{g}^{\alpha}:=h\left(\gamma^{\alpha}\right) .
$$

The extensor field $h$ may be used to introduce on $T^{*} M$ the following extensor fields

$$
g=h^{-1 \dagger} h^{-1}, \ldots g^{-1}=h h^{\dagger} .
$$

Of course, we have7

$$
g\left(\mathfrak{g}^{\alpha}\right) \cdot\left(\mathfrak{g}^{\beta}\right)=h^{-1}\left(\mathfrak{g}^{\alpha}\right) \cdot h_{\dot{\mathfrak{g}}}^{-1}\left(\mathfrak{g}^{\beta}\right)=\gamma_{\dot{\mathfrak{g}}}^{\alpha} \cdot \gamma^{\beta}=\eta^{\alpha \beta} .
$$

We can think of $g$ as the associated extensor field a tensor field $g \in \sec T_{2}^{0} M$

$$
\mathbf{g}:=\eta_{\alpha \beta} \mathfrak{g}^{\alpha} \otimes \mathfrak{g}^{\beta} .
$$

Moreover, the extensor field $g^{-1}$ is associated to the field $\mathrm{g} \in \sec T_{0}^{2} M$ such that

$$
\mathrm{g}=\eta^{\alpha \beta} \mathfrak{e}_{\alpha} \otimes \mathfrak{e}_{\beta},
$$

with $\left\{\mathfrak{e}_{\alpha}\right\}, \mathfrak{e}_{\alpha} \in \sec T M$, the dual basis of $\left\{\mathfrak{g}^{\alpha}\right\}$, i.e., $\mathfrak{g}^{\alpha}\left(\mathfrak{e}_{\beta}\right)=\delta_{\beta}^{\alpha}$.

Moreover, in our theory each nontrivial gravitational field configuration, i.e., one from which not all the $\mathfrak{g}^{\alpha}$ are exact differentials can be interpreted as generating an effective Lorentzian spacetime $\&^{8}\left(M \simeq \mathbb{R}^{4}, \boldsymbol{g}, D, \tau_{g}, \uparrow\right)$ where $D$ is the Levi-Civita connection of $\boldsymbol{g}$ interpreted as a Lorentzian metric on $M$ and such that the $\mathfrak{g}^{\alpha}$ satisfy Maxwell like field equations (which follows from the variational principle [2]). and which are equivalent to Einstein equation for the gravitational field in General Relativity (GR).

Remark 1 Before proceeding we recall that given $g \in \sec T_{0}^{2} M$ we can construct the Clifford bundle $\mathcal{C} \ell(M, \mathrm{~g})$. The Clifford product, the left and right contractions and the Hodge star operators defined by $g$ can be easily expressed in the Clifford bundle $\mathcal{C} \ell(M, \mathrm{~g})$ through the Golden formula (see, e.g., [Q]). Indeed, if $*$ denotes either the exterior product $(\wedge)$, or the $\mathrm{g}-$ scalar product, or the $\mathrm{g}$-contracted products $(\underset{\mathrm{g}}{(}, \mathrm{L}, \stackrel{\mathrm{g}}{)}$ or the $\mathrm{g}-$ Clifford product and analogously for * we have for any $X, Y \in \sec \bigwedge T^{*} M$ that

$$
\underline{h}^{-1}(X \underset{\mathrm{g}}{* Y})=\underline{h}^{-1}(X) \underset{\dot{\mathrm{g}}}{*} \underline{h}^{-1}(Y) .
$$

\footnotetext{
${ }^{7}$ We have for $A, B \in \bigwedge^{1} T^{*} M \hookrightarrow \sec \mathcal{C} \ell(M, \stackrel{\mathrm{g}}{)})$ that $A_{\dot{\mathrm{g}}} B:=\mathrm{g}(A, B)$.

${ }^{8}$ Or by an effective teleparallel spacetime, see [7, the arXiv version.
} 
Moreover the relation between the Hodge star operators $\underset{\mathrm{g}}{\star} \underset{\dot{\mathrm{g}}}{\text { and }} \underset{\text { is }}{\star}$

$$
\stackrel{\star}{\mathrm{g}}=\underline{h}^{-1 \dagger} \underset{\stackrel{\mathrm{g}}{h}}{\star},
$$

where in the above formulas $\underline{h}$ means the exterior power extension of $h$.

Recall next that it is a physicist dream to construct a quantum theory for the gravitational field, where the quanta of the field are the so called gravitons. In such (yet to be constructed) theory the gravitational field is supposed to be represented by a distribution valued symmetric field operator acting on the Hilbert space of the system. Classically that field is represented by a symmetric tensor (distribution)

$$
\mathbf{h}=\mathbf{h}_{\alpha \beta} \vartheta^{a} \otimes \vartheta^{\beta} \in \sec T_{0}^{2} M,
$$

where9 $\vartheta^{\mu}:=d x^{\mu} \in \sec \bigwedge^{1} T^{*} M \hookrightarrow \sec \mathcal{C} \ell(M, \stackrel{\circ}{\mathrm{g}})$, with $\left\{x^{\mu}\right\}$ arbitrary coordinates covering $U \subset M$. Such a general field, as it is well known [1, 11] carries a direct sum of irreducible representations of the Lorentz group, one carrying spin two, one carrying spin one and two carrying spin zero. Now, consider the tensor field $\mathbf{h}^{\prime} \in \sec T_{1}^{1} M$

$$
\mathbf{h}^{\prime}=\phi_{\beta}^{\alpha} \frac{\partial}{\partial x^{\alpha}} \otimes d x^{\beta}
$$

If we impose that $\operatorname{divh}^{\prime}=0$, i.e., the restriction 10

$$
\stackrel{\circ}{D}_{\alpha} \phi_{\beta}^{\alpha}=0,
$$

(where $\phi_{\beta}^{\alpha}:=\stackrel{\circ}{g}^{\alpha \kappa} \phi_{\kappa \beta}, \stackrel{\circ}{g}=\stackrel{\circ}{g}_{\alpha \beta} \vartheta^{a} \otimes \vartheta^{\beta}$ ) then the field $\mathbf{h}$ carries only the irreducible representations with spin two and one with spin zero of the Lorentz group. This restriction is the one appropriate for the description of gravitons with non null mass $m$.

Next we introduce the main purpose of this paper, which is to investigate (using the Clifford bundle formalism) the consequences of the ansatz

$$
\begin{aligned}
& \mathbf{h}^{\prime}=h=\phi_{\beta}^{\alpha} \frac{\partial}{\partial x^{\alpha}} \otimes d x^{\beta}=h_{\beta}^{\alpha} \frac{\partial}{\partial \mathrm{x}^{\alpha}} \otimes d \mathrm{x}^{\beta} \\
& \mathfrak{g}^{\alpha}:=h_{\beta}^{\alpha} \gamma^{\beta} .
\end{aligned}
$$

We then show how to derive from our Lagrangian theory the exact wave equation for the graviton field and we obtain a reliable conservation law for the energy-momentum tensor of the gravitational plus the matter fields in Minkowski spacetime.

\footnotetext{
${ }^{9}$ Given arbitrary coordinate functions $\left\{\boldsymbol{x}^{\mu}\right\}$ covering $U \subset M$ with coordinates $\left\{x^{\mu}\right\}$ such that $\mathbf{x}^{\mu}(\mathfrak{e})=x^{\mu}$, we write, as usual, $\left\{\frac{\partial}{\partial x^{\mu}}\right\}$ for the coordinate tangent vector fields and $\left\{d x^{\mu}\right\}$ for the coordinate cotangent covectors.

${ }^{10}$ In [7] we show explicitly how to determine the extensor field $h$ once $g$ is known in a given basis.
} 
We also ask the question: does Eq.(10) fix any gauge condition for the field $\mathbf{g}$ of the effective Lorentzian spacetime structure $\left(M \simeq \mathbb{R}^{4}, \boldsymbol{g}, D, \tau_{\mathrm{g}}, \uparrow\right)$ that is a well defined functional of the field $\mathbf{h}$ in our theory? We show that no gauge condition is fixed a priory, as it is the case in GR. Thus, writing $g=g^{\alpha \beta} \frac{\partial}{\partial x^{\alpha}} \otimes \frac{\partial}{\partial x^{\beta}}$ we do not need, e.g., to fix in our theory Logunov gauge condition

$$
\stackrel{\circ}{D}_{\gamma}\left(\sqrt{-\operatorname{det} g} g^{\gamma \kappa}\right)=0,
$$

which, is indeed a result of a postulate in Logunov's theory [3, 4. Since Logunov thinks that Eq. (12) is very important, since according to him it fixes a unique solution of Einstein equation: 11 once a matter distribution and a coordinate chart are given, thus eliminating (possible) ambiguities in predictions of experiments. We discuss briefly this issue.

\section{The Wave Equation for the $\mathfrak{g}^{\alpha}$}

We recall that the dynamics of the fields $\mathfrak{g}^{\alpha}$ in a region of $M$ is given by

$$
\mathcal{L}=\mathcal{L}_{g}+\mathcal{L}_{m},
$$

where $\mathcal{L}_{m}$ is the Lagrangian density of the matter fields and

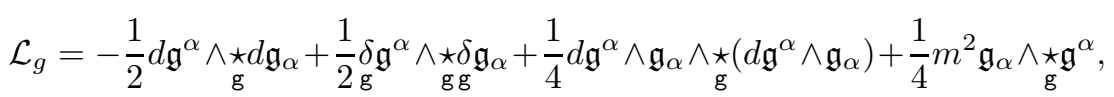

is invariant ( modulo an exact form.) under local Lorentz transformations 12 , which is a kind of gauge freedom, a crucial ingredient of our theory, as showed in 7].

The $\mathfrak{g}^{\alpha}$ couple universally to the matter fields in such a way that the energy momentum 1-form of the matter fields are given by

$$
\underset{\mathrm{g}}{\star \mathfrak{T}^{\alpha}}=\frac{\partial \mathcal{L}_{m}}{\partial \mathfrak{g}_{\alpha}} .
$$

Each one of the fields $\mathfrak{g}^{\alpha}$ in Eq.(14) resembles a potential of an electromagnetic field. Indeed, the first term is of the Yang-Mills type, the second term is a kind of gauge fixing term (analogous to the Lorenz condition for the gauge potential of the electromagnetic potential), and more important, the condition given by Eq. (10) is equivalent to

$$
\underset{\substack{0 \\ \mathfrak{g}}}{\delta \mathfrak{g}^{\alpha}}=0 .
$$

\footnotetext{
${ }^{11}$ Even for the case of a zero mass graviton.

${ }^{12}$ We observe that the various coefficients in Eq. 14 have been selected in order for $\mathcal{L}_{g}^{M}$ to be invariant under arbitrary local Lorentz transformations. This means, as the reader may verify that under the transformation $\mathfrak{g}^{\mathbf{a}} \mapsto u \mathfrak{g}^{\mathbf{a}} u^{-1}, u \in \sec \operatorname{Spin}_{1,3}^{e}(M, \mathrm{~g}) \hookrightarrow \sec \mathcal{C} \ell(M, \mathrm{~g})$, $\mathcal{L}_{g}^{M}$ is invariant modulo an exact form.
} 
Indeed, given the coordinates functions $\left\{\mathbf{x}^{\mu}\right\}$ for $U \subset M, \mathbf{x}^{\mu}(\mathfrak{e})=\mathrm{x}^{\mu}\left(\mathfrak{g}^{\alpha}=h_{\beta}^{\alpha} \gamma^{\beta}\right.$ and $\left.\stackrel{\circ}{\mathbf{g}}=\eta_{\alpha \beta} \gamma^{\alpha} \otimes \gamma^{\beta}\right)$ it is 13

$$
\begin{aligned}
\dot{\mathrm{g}}_{\dot{\mathrm{g}}} \mathfrak{g}^{\alpha} & \left.=-\phi_{\overrightarrow{\mathrm{g}}} \mathfrak{g}^{\alpha}=-\gamma^{\kappa}\right\lrcorner\left(\stackrel{D}{\grave{\mathrm{g}}}_{\frac{\partial}{\partial \mathrm{x}^{\kappa}}}\left(h_{\beta}^{\alpha} \gamma^{\beta}\right)\right) \\
& =-\left(\stackrel{\circ}{D}_{\kappa} h_{\beta}^{\alpha}\right) \eta^{\kappa \beta}=\partial_{\kappa} h_{\lambda}^{\kappa}=0
\end{aligned}
$$

Moreover, take notice that in general

$$
{ }_{\mathrm{g}} \mathfrak{g}^{\alpha}=-\partial \underset{\dot{\mathfrak{g}}}{\lrcorner} \mathfrak{g}^{\alpha} \neq 0,
$$

where for any $A_{p} \in \sec \bigwedge^{p} T^{*} M \hookrightarrow \sec \mathcal{C} \ell(M, \stackrel{\mathrm{g}}{\mathrm{g}})$, it is $\underset{\mathrm{g}}{\delta} A_{p}=\vartheta_{\mathrm{g}}^{\kappa} \underset{\mathrm{g}}{\perp} \frac{\partial}{\partial x^{\kappa}} A_{p}$ and $\left.\oint_{\mathrm{g}} A_{p}:=-\vartheta_{\mathrm{g}}^{\kappa}\right\lrcorner \frac{\partial}{\partial x^{\kappa}} A_{p}$.

Also, the third term in the Lagrangian density is a self-interacting term, which is proportional to the square of the total 'vorticity' $\Omega=d \mathfrak{g}^{\alpha} \wedge \mathfrak{g}_{\alpha}$ associated to the 1 -form fields $\mathfrak{g}^{\alpha}$. This shows that in the Lagrangian density the $\mathfrak{g}^{\alpha}$ does not couple with the energy-momentum tensor of the gravitational field 14 , which according to the Lagrangian formalism is given by $\frac{\partial \mathcal{L}_{g}}{\partial \mathfrak{g}^{\alpha}}$. We finally recall that as showed in details in [7] $\mathcal{L}_{g}$ differs (when the graviton mass is null) from the Einstein-Hilbert Lagrangian by an exact differential.

Also, as showed in details. e.g., in [2] variation of $\int \mathcal{L}_{g}$ produces the following equations of motion

$$
d \underset{\mathrm{g}}{\star} \mathcal{S}^{\alpha}+\underset{\mathrm{g}}{\star \mathrm{t}^{\alpha}}+\frac{1}{2} m_{\mathrm{g}}^{2} \underset{\mathrm{g}}{\mathrm{g}} \mathrm{g}^{\alpha}=-\underset{\mathrm{g}}{\star} \mathfrak{T}^{\alpha},
$$

with $\underset{\mathrm{g}}{\star \mathrm{t}^{\kappa}} \in \sec \bigwedge^{3} T^{*} M \hookrightarrow \mathcal{C} \ell\left(T^{*} M, \mathrm{~g}\right)$ and $\underset{\mathrm{g}}{\star \mathcal{S}^{\kappa}} \in \sec \bigwedge^{2} T^{*} M \hookrightarrow \mathcal{C} \ell\left(T^{*} M, \mathrm{~g}\right)$ given by

$$
\begin{aligned}
& \left.\underset{\mathrm{g}}{\star \mathfrak{t}^{\kappa}}=\frac{\partial \mathcal{L}_{g}}{\partial \mathfrak{g}_{\kappa}}=\frac{1}{2}\left[\left(\mathfrak{g}_{\kappa}\right\lrcorner d \mathfrak{g}_{\mathrm{g}}^{\alpha}\right) \wedge \underset{\mathrm{g}}{\star} d \mathfrak{g}_{\alpha}-d \mathfrak{g}^{\alpha} \wedge\left(\mathfrak{g}_{\kappa} \underset{\mathrm{g}}{\lrcorner} \underset{\mathrm{g}}{\star} d \mathfrak{g}_{\alpha}\right)\right]
\end{aligned}
$$

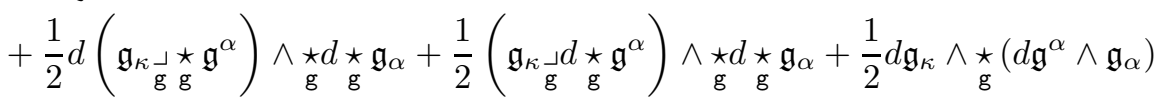

$$
\begin{aligned}
& -\frac{1}{4} d \mathfrak{g}^{\alpha} \wedge \mathfrak{g}_{\alpha} \wedge\left[\mathfrak{g}_{\kappa} \underset{\mathrm{g}}{\lrcorner} \underset{\mathrm{g}}{\star}\left(d \mathfrak{g}^{\iota} \wedge \mathfrak{g}_{\iota}\right)\right]-\frac{1}{4}\left[\mathfrak{g}_{\kappa} \underset{\mathrm{g}}{\lrcorner}\left(d \mathfrak{g}^{\iota} \wedge \mathfrak{g}_{\iota}\right)\right] \wedge \underset{\mathrm{g}}{\star}\left(d \mathfrak{g}^{\alpha} \wedge \mathfrak{g}_{\alpha}\right), \\
& \underset{\mathrm{g}}{\star} \mathcal{S}^{\kappa}=\frac{\partial \mathcal{L}_{g}}{\partial d \mathfrak{g}_{\kappa}}=-\mathfrak{g}^{\alpha} \wedge \underset{\mathrm{g}}{\star}\left(d \mathfrak{g}_{\alpha} \wedge \mathfrak{g}_{\kappa}\right)+\frac{1}{2} \mathfrak{g}_{\kappa} \wedge \underset{\mathrm{g}}{\star}\left(d \mathfrak{g}^{\alpha} \wedge \mathfrak{g}_{\alpha}\right) .
\end{aligned}
$$

\footnotetext{
${ }^{13}$ As usual we put $\stackrel{\circ}{D} \frac{\partial}{\partial x^{\kappa}}\left(\mathbf{h}_{\beta}^{\alpha} d x^{\beta} \otimes \frac{\partial}{\partial x^{\alpha}}\right):=\left(\stackrel{\circ}{D}_{\kappa} \mathbf{h}_{\beta}^{\alpha}\right) d x^{\beta} \otimes \frac{\partial}{\partial x^{\alpha}}$. Moreover, we have for any $A_{p} \in \sec \bigwedge^{1} T^{*} M \hookrightarrow \sec \mathcal{C} \ell\left(T^{*} M, \stackrel{\mathrm{g}}{\mathrm{g}}\right)$ that the action of the Dirac like operators $\phi$ and $\boldsymbol{\partial}$ are: $\phi A_{p}:=\vartheta^{\alpha}{ }_{\overrightarrow{\mathrm{g}}}\left(D_{e_{\alpha}} A_{p}\right)+\vartheta^{\alpha} \wedge\left(D_{e_{\alpha}} A_{p}\right)$ and $\boldsymbol{\partial} A_{p}:=\vartheta^{\alpha} \underset{\mathrm{g}}{\lrcorner}\left(D_{e_{\alpha}} A_{p}\right)+\vartheta^{\alpha} \wedge\left(D_{e_{\alpha}} A_{p}\right)$. For more details see 9 [14.

${ }^{14}$ On this respect see the discussion of [13].
} 
For what follows we need also the following equivalent expression for the $\star \mathcal{S}^{\kappa}$ obtained, e.g., in [8],

$$
\left.\left.\left.\underset{\mathrm{g}}{\star} \mathcal{S}^{\kappa}=\frac{1}{2} \underset{\mathrm{g}}{\star}\left[-\left(\mathfrak{g}_{\alpha}\right\lrcorner d \mathfrak{g}_{\mathrm{g}}^{\alpha}\right) \wedge \mathfrak{g}^{\kappa}-\left(\mathfrak{g}_{\mathrm{g}}^{\alpha}\right\lrcorner d \mathfrak{g}_{\alpha}\right) \wedge \mathfrak{g}^{\kappa}+\left(\mathfrak{g}_{\mathrm{g}}^{\kappa}\right\lrcorner d \mathfrak{g}^{\alpha}\right) \wedge \mathfrak{g}_{\alpha}-d \mathfrak{g}^{\kappa}\right] .
$$

We write moreover

$$
\underset{\mathrm{g}}{\star} \mathcal{S}^{\kappa}=-\frac{1}{2} \underset{\mathrm{g}}{\star} d \mathfrak{g}^{\kappa}+\underset{\mathrm{g}}{\star \mathfrak{K}^{\kappa}}
$$

and insert this result in Eq.(19) obtaining:

$$
-\frac{1}{2} d \underset{\mathrm{g}}{\star} d \mathfrak{g}^{\kappa}+\frac{1}{2} m_{\mathrm{g}}^{2} \underset{\mathrm{g}}{\star} \mathfrak{g}^{\kappa}=-\underset{\mathrm{g}}{\star}\left(\mathfrak{t}^{\kappa}+\mathfrak{T}^{\kappa}+\underset{\mathrm{g}}{\star^{-1}} d \underset{\mathrm{g}}{\star} \mathfrak{K}^{\kappa}\right)
$$

Before proceeding we recall that we have the conservation law

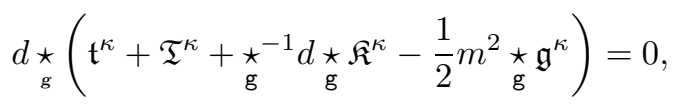

We now add the term $-\frac{1}{2} d \delta_{\mathrm{g}} \mathfrak{g}^{\kappa}$ to both members Eq.(24) and next apply the operator $\underset{\mathrm{g}}{\star^{-1}}$ to both sides of that equation, thus obtaining the equivalent equation:

$$
-\frac{1}{2} \delta d \mathfrak{g}^{\kappa}-\frac{1}{2} d \delta \mathfrak{g}^{\kappa}+\frac{1}{2} m^{2} \mathfrak{g}^{\kappa}=-\left(\mathfrak{t}^{\kappa}+\mathfrak{T}^{\kappa}+\underset{\mathrm{g}}{\delta \mathfrak{K}^{\kappa}}+\frac{1}{2} d \delta \mathfrak{g}^{\kappa}\right)
$$

We now recall the definition of the Hodge D'Alembertian, which in the Clifford bundle formalism is the square of the Dirac operator $\boldsymbol{\partial}:=\vartheta^{\alpha} D_{e_{\alpha}}$ acting on sections of the Clifford bundle $\mathcal{C} \ell\left(T^{*} M, \stackrel{\mathrm{g}}{)}\right)[6$, i.e.,

$$
\stackrel{\mathrm{g}}{\diamond \mathfrak{g}^{\kappa}}:=\underset{\mathrm{g}}{-\underset{\mathrm{g}}{\delta} d-d \delta} \mathfrak{g}^{\kappa}=\partial^{2} \mathfrak{g}^{\kappa}
$$

and recall moreover the following nontrivial decomposition [6] of $\phi^{2}$,

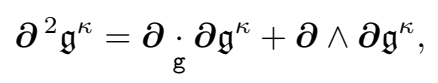

where $\stackrel{\mathrm{g}}{\square}:=\boldsymbol{\partial} \cdot \boldsymbol{\partial}$ is the covariant D'Alembertian and $\boldsymbol{\partial} \wedge \boldsymbol{\partial}$ is the Ricci operator associated to the Levi-Civita connection $D$ of $\mathrm{g}$. Moreover, we have

$$
\boldsymbol{\partial} \wedge \partial \mathfrak{g}_{\kappa}=\mathcal{R}_{\kappa}=R_{\kappa \iota} \vartheta^{\iota},
$$

where $\mathcal{R}_{\kappa} \in \sec \bigwedge^{1} T^{*} M \hookrightarrow \mathcal{C} \ell\left(T^{*} M, \mathrm{~g}\right)$ are the Ricci 1-form fields and $R_{\kappa \iota}$ are the components of the Ricci tensor. 
This permits us to rewrite Eq.(26) as

$$
\frac{1}{2} \stackrel{\mathrm{g}}{\square} \mathfrak{g}^{\kappa}+\frac{1}{2} m^{2} \mathfrak{g}^{\kappa}=-\mathfrak{T}^{\kappa}-\mathfrak{t}^{\kappa}-\delta_{\mathrm{g}} \mathfrak{K}^{\kappa}-\frac{1}{2} d \delta \mathfrak{g}_{\mathrm{g}}^{\kappa}-\mathcal{R}^{\kappa}=-\mathbf{T}^{\kappa} .
$$

Thus, writing (recall Eq.(11))

$$
\begin{gathered}
\phi_{\iota}^{\kappa}:=h_{\mu}^{\kappa} \frac{\partial \mathrm{x}^{\mu}}{\partial x^{\iota}}, \\
\mathfrak{g}^{\kappa}=\phi_{\iota}^{\kappa} d x^{\iota}, \mathbf{T}^{\kappa}=\mathbf{T}_{\iota}^{\kappa} d x^{\iota},
\end{gathered}
$$

and taking into account that $[6]$

$$
\stackrel{\mathrm{g}}{\square} \mathfrak{g}^{\kappa}=\left(g^{\alpha \beta} D_{\alpha} D_{\beta} \phi_{\iota}^{\kappa}\right) d x^{\iota}
$$

we get from Eq.(30)

$$
\phi_{\kappa}^{\alpha} \stackrel{\circ}{g}^{\kappa \iota} \phi_{\iota}^{\beta} D_{\alpha} D_{\beta} \phi_{\iota}^{\kappa}+m^{2} \phi_{\iota}^{\kappa}=-2 \mathbf{T}_{\iota}^{\kappa},
$$

which is in our theory a possible form for the (covariant) equation for the (nonlinear) graviton field on Minkowski spacetime. The last statement follows because $D_{\alpha}$ can be easily be expressed in terms of the $\stackrel{\circ}{D}_{\alpha}$ using the formulas of the Appendix.

Remark 2 We can immediately write from Eq.(24) that

$$
\underset{\mathrm{g}}{\delta}\left(\mathfrak{t}^{\kappa}+\mathfrak{T}^{\kappa}+\underset{\mathrm{g}}{\delta \mathfrak{K}^{\kappa}}-\frac{1}{2} m^{2} \mathfrak{g}^{\kappa}\right)=0
$$

Remark 3 Eq. 35. express as we already anticipated a reliable conservation law for the total energy-momentum of the matter plus the gravitational field. However, take notice that in GR this result depends on the fixing of a cotetrad basis and changing it by a local Lorentz transformation changes accordingly the energy-momentum tensor of the gravitational field. In fact this last result has already been known since the wort15 of Møller [12].

Remark 4 Moreover, we see that imposing the Lorenz type gauge $\underset{\mathrm{g}}{\delta} \mathfrak{g}^{\kappa}=0$ to the dynamic gravitational fields amounts to exclude the graviton energy density from the conservation law.

\footnotetext{
${ }^{15}$ Which however did not use the present crystal clear formalism.
} 


\section{Which Gauge to Use for $g$ in the Effective Lorentzian Spacetime?}

We already recalled that our Lagrangian density differs for the Einstein-Hilbert Lagrangian by an exact form. But it can also be written as:

$$
\mathcal{L}_{g}=-\frac{1}{2}\left(d \mathfrak{g}_{\alpha} \wedge \mathfrak{g}^{\beta}\right) \wedge \underset{\mathrm{g}}{\star}\left(d \mathfrak{g}_{\beta} \wedge \mathfrak{g}^{\alpha}\right)+\frac{1}{4} d \mathfrak{g}_{\alpha} \wedge \mathfrak{g}^{\alpha} \wedge \underset{\mathrm{g}}{\star}\left(d \mathfrak{g}_{\beta} \wedge \mathfrak{g}^{\beta}\right)
$$

which can be shown [6, 2] to be equivalent (modulus an exact differential) to

$$
\mathcal{L}_{g}=-\frac{1}{2}\left(d \vartheta_{\alpha} \wedge \vartheta^{\beta}\right) \wedge \underset{\mathrm{g}}{\star}\left(d \vartheta_{\beta} \wedge \vartheta^{\alpha}\right)+\frac{1}{4} d \vartheta_{\alpha} \wedge \vartheta^{\alpha} \wedge \underset{\mathrm{g}}{\star}\left(d \vartheta_{\beta} \wedge \vartheta^{\beta}\right)
$$

where $\left\{\vartheta^{\alpha}\right\}$ is an an arbitrary coframe basis, not necessarily $\boldsymbol{g}$ orthonormal, and where $\vartheta_{\alpha}:=g_{\alpha \beta} \vartheta^{\beta}$

This permit us 2] to obtain an equation analogous to Eq.(19), i.e.,

$$
d \underset{\mathrm{g}}{\star} S^{\alpha}+\underset{\mathrm{g}}{\star t^{\alpha}}+\frac{1}{2} m_{\mathrm{g}}^{2} \star \vartheta^{\alpha}=-\underset{\mathrm{g}}{\star} T^{\alpha} .
$$

So, let us examine the structure of Eq. (38) in a coordinate basis $\left\{\vartheta^{\mu}=d x^{\mu}\right\}$. We immediately see that a conservation law (distinct from the previous one established above) 16 in the effective Lorentzian spacetime structure (excluding the energy associated with the graviton mass) exists for $\underset{\mathrm{g}}{\star}\left(T^{\mu}+t^{\mu}\right)$ if

$$
\underset{\mathrm{g}}{\delta} \vartheta^{\mu}=0
$$

This of course, implies that

$$
\diamond x^{\mu}=-\underset{\mathrm{g}}{d \delta} x_{\mathrm{g}}^{\mu}-\delta^{\mu}=0,
$$

i.e., the coordinates must be harmonic.

Now, if

$$
D_{\frac{\partial}{\partial x^{\nu}}} \frac{\partial}{\partial x^{\mu}}=\Gamma_{\nu \mu}^{\rho} \frac{\partial}{\partial x^{\rho}}, \quad D_{\frac{\partial}{\partial x^{\nu}}} \vartheta^{\mu}=-\Gamma_{\nu \rho}^{\mu} \vartheta^{\rho}
$$

we have

$$
\begin{aligned}
& \underset{\mathrm{g}}{\delta \vartheta^{\mu}}=-\vartheta^{\nu}{ }_{\mathrm{g}} D_{\frac{\partial}{\partial \mathrm{x}^{\nu}}} \vartheta^{\mu}=\vartheta^{\nu}{ }_{\mathrm{g}}\left(\Gamma_{\nu \alpha}^{\mu} \vartheta^{\alpha}\right)=\Gamma_{\nu \alpha}^{\mu} g^{\nu \alpha}=0, \\
& \diamond x^{\mu}=0 \Rightarrow \Gamma_{\nu \alpha}^{\mu} g^{\nu \alpha}=0
\end{aligned}
$$

\footnotetext{
${ }^{16}$ Recall that in GR Eq. 38 implies in a pseudo conservation law because in that theory (without a Minkowski spacetime interpretation, as here) $S^{\alpha}$ are expressed in terms of connection -forms of the Levi-Civita connection of $g$ and thus are not indexed forms. Details may be found in $[8]$.
} 
Now, if $\Gamma_{\nu \alpha}^{\mu} g^{\nu \alpha}=0$ we have

$$
\begin{aligned}
D_{\mu}\left(\sqrt{-\operatorname{det} \boldsymbol{g}} g^{\mu \nu}\right) & =\frac{\partial}{\partial x^{\mu}}\left(\sqrt{-\operatorname{det} \boldsymbol{g}} g^{\mu \nu}\right)+\Gamma_{\mu \alpha}^{\nu} \sqrt{-\operatorname{det} \boldsymbol{g}} g^{\mu \alpha} \\
& =\frac{\partial}{\partial x^{\mu}}\left(\sqrt{-\operatorname{det} \boldsymbol{g}} g^{\mu \nu}\right) \\
& =\frac{\partial}{\partial x^{\mu}}\left(\sqrt{-\operatorname{det} \boldsymbol{g}} g^{\mu \nu}\right)+\stackrel{\circ}{\Gamma}_{\mu \alpha}^{\nu} \sqrt{-\operatorname{det} \boldsymbol{g}} g^{\mu \alpha}-\stackrel{\circ}{\Gamma}_{\mu \alpha}^{\nu} \sqrt{-\operatorname{det} \boldsymbol{g}} g^{\mu \alpha} \\
& =\stackrel{\circ}{D}_{\mu}\left(\sqrt{-\operatorname{det} \boldsymbol{g}} g^{\mu \nu}\right)-\stackrel{\circ}{\Gamma}_{\mu \alpha}^{\nu} \sqrt{-\operatorname{det} \boldsymbol{g}} g^{\mu \alpha}
\end{aligned}
$$

and since $D_{\mu}\left(\sqrt{-\operatorname{det} g} g^{\mu \nu}\right)=0$ we get that

$$
\diamond x^{\mu}=0 \Rightarrow \stackrel{\circ}{D}_{\mu}\left(\sqrt{-\operatorname{det} \boldsymbol{g}} g^{\mu \nu}\right)=\stackrel{\circ}{\Gamma}_{\mu \alpha}^{\nu} \sqrt{-\operatorname{det} \boldsymbol{g}} g^{\mu \alpha}
$$

In particular in a coordinate basis $\left\{\gamma^{\mu}=d \mathrm{x}^{\mu}\right\}$, where $\left\{\mathrm{x}^{\mu}\right\}$ are global coordinates for $M$ in Einstein-Lorentz-Poincaré gauge 17 where the connection coefficients are null we have

$$
\diamond \mathrm{x}^{\mu}=0 \Rightarrow \stackrel{\circ}{D}_{\mu}\left(\sqrt{-\operatorname{det} g} g^{\mu \nu}\right)=\frac{\partial}{\partial \mathrm{x}^{\mu}}\left(\sqrt{-\operatorname{det} \boldsymbol{g}} g^{\mu \nu}\right)=0 .
$$

But since $\sqrt{-\operatorname{det}(\mathbf{g})} \mathrm{g}^{\mu \nu}$ are the components of the tensor density $\mathfrak{G} \in \sec T_{0}^{2} M \otimes$ $\bigwedge^{4} T^{*} M$, which on an arbitrary basis is written as

$$
\mathfrak{G}=\sqrt{-\operatorname{det} g} g^{\mu \nu} \frac{\partial}{\partial x^{\mu}} \otimes \frac{\partial}{\partial x^{\nu}} \otimes d x^{0} \wedge d x^{1} \wedge d x^{2} \wedge d x^{3}
$$

we arrive at the conclusion that

$$
\stackrel{\circ}{D}_{\mu}\left(\sqrt{-\operatorname{det} g} g^{\mu \nu}\right)=0,
$$

which is Logunov gauge condition! Note that this also implies that on any arbitrary basis we must have

$$
\stackrel{\circ}{\Gamma}_{\mu \alpha}^{\nu} g^{\mu \alpha}=0 .
$$

Also, for arbitrary non harmonic coordinates functions $\left\{x^{\mu}\right\}$ we get $\left(D^{\circ} \frac{\partial}{\partial x^{v}} \frac{\partial}{\partial x^{\mu}}=\right.$ $\left.\stackrel{\circ}{\Gamma}_{\mu \nu}^{\kappa} \frac{\partial}{\partial x^{\kappa}}, D_{\frac{\partial}{\partial x^{\nu}}} \frac{\partial}{\partial x^{\mu}}=\Gamma_{\mu \nu}^{\kappa} \frac{\partial}{\partial x^{\kappa}}\right)$ that

$$
\diamond x^{\mu}=-\Gamma_{\alpha \nu}^{\mu} g^{\alpha \nu}=-\stackrel{\circ}{\Gamma}_{\alpha \nu}^{\mu} g^{\alpha \nu}-K_{\alpha \nu}^{\mu} g^{\alpha \nu} \neq 0 .
$$

Remark $\mathbf{5}$ Now, given the Logunov gauge condition, it does not imply that the coordinates are harmonic ones, for we have (using the formulas in the Appendix) that

$$
\begin{aligned}
\stackrel{\circ}{D}_{\mu}\left(\sqrt{-\operatorname{det} g} g^{\mu \nu}\right) & =D_{\mu}\left(\sqrt{-\operatorname{det} g} g^{\mu \nu}\right)+K_{\mu \kappa}^{\nu} \sqrt{-\operatorname{det} g} g^{\mu \kappa} \\
& =K_{\mu \kappa}^{\nu} \sqrt{-\operatorname{det}\left(g_{\mu \nu}\right)} g^{\mu \kappa}
\end{aligned}
$$

\footnotetext{
${ }^{17}$ Recall that the energy-momentum conservation law of any Lorentz invariant field theory is unambiguously formulated global coordinates in Einstein-Lorentz-Poincaré gauge.
} 
and thus

$$
\stackrel{\circ}{D}_{\mu}\left(\sqrt{-\operatorname{det} g} g^{\mu \nu}\right)=0 \Rightarrow K_{\mu \kappa}^{\nu} \sqrt{-\operatorname{det} g} g^{\mu \kappa}=0
$$

and we see that under those condition 18 the allowed coordinate functions of Logunov theory must always satisfy the constraint:

$$
\diamond x^{\mu}=-\Gamma_{\alpha \nu}^{\mu} g^{\alpha \nu}=-\stackrel{\circ}{\Gamma}_{\alpha \nu}^{\mu} g^{\alpha \nu}
$$

Remark 6 As an additional remark we comment that Logunov [3, 4] imposed Eq. (12) as a gauge condition in his theory because he postulated (differently from what is the case here, see below) that the relation between $g_{L}^{\alpha \beta}$ and the gravitational field $\mathbf{h}^{\alpha \beta}$ is given by

$$
\underset{L}{\varkappa} g^{\alpha \beta}:=\stackrel{\circ}{g} \alpha \beta+\mathbf{h}^{\alpha \beta} .
$$

Now, the second member of Eq. (51) implies immediately taking into account Eq.(10) that $\stackrel{\circ}{D}_{\alpha}\left(\varkappa_{L} g^{\alpha \beta}\right)=0$ and thus from the second line in Eq. (77) (in Appendix) we get that $\stackrel{\circ}{D}_{\alpha}\left(\sqrt{-\operatorname{det} g g^{\alpha \beta}}\right)=0$.

Remark 7 In our theory, defining $\left\{\vartheta_{\mu}\right\}$ as the reciprocal basis of $\left\{\vartheta^{\mu}\right\}$ relative to $g$, i.e., $g\left(\vartheta_{\mu}, \vartheta^{\nu}\right)=\delta_{\mu}^{\nu}$ we can write directly from Eq.(??) that

\section{Remark 8}

$$
\begin{aligned}
\mathbf{g} & =\eta^{\alpha \beta} h\left(\gamma_{\alpha}\right) \otimes h\left(\gamma_{\beta}\right) \\
& =g^{\kappa \iota} \vartheta_{\kappa} \otimes \vartheta_{\iota},
\end{aligned}
$$

and clearly, differently from Logunov's theory we have:

$$
\varkappa g^{\alpha \beta} \neq \underset{L}{\varkappa g^{\alpha \beta}}
$$

from where it follows that there is no need to impose a priory any gauge condition (as it is the case in GR) for the "metric" field $\mathbf{g}$ of the effective Lorentzian spacetime.

\section{Conclusions}

We showed that if gravitation is to be described by a massive graviton field living in Minkowski spacetime which is represented by a symmetric tensor field $\mathbf{h}$ carrying the representations of spin two and zero of the Lorentz group and thus satisfying the gauge condition given by Eq.(10) then the effective Lorentzian spacetime structure that represents the gravitational field (under the ansatz

\footnotetext{
${ }^{18}$ The ones in Logunov theory.
} 
given by Eq.(11) ) of a given energy-momentum distribution is such that the field $g$ solving the effective Einstein-Hilbert equation (with cosmological constant) as it is the case in GR - does not need to satisfy a priory any fixed gauge. We showed moreover that the Logunov gauge condition $\stackrel{\circ}{D}_{\gamma}\left(\sqrt{-\operatorname{det} g} g^{\gamma \kappa}\right)=0$ ( which in his theory is indeed a postulate) does not hold in general in our theory without ad hoc hypothesis. If such a gauge is postulated it implies that the allowed coordinate functions to be the ones satisfying Eq.(50), i.e., the theory is not covariant. Moreover, we proved that the imposition of the Lorenz type gauge gauge $\delta \mathfrak{g}^{\kappa}=0$ to the dynamic gravitational fields amounts to exclude the graviton energy density from the energy-momentum conservation law, something that eventually may shed some light on the problem of the dark energy.

Logunov thought that the importance of the condition $\stackrel{\circ}{D}_{\gamma}\left(\sqrt{-\operatorname{det} g} g^{\gamma \kappa}\right)=$ 0 in determining the effective Lorentzian spacetime generated by an energymomentum distribution can be seem from the following example 3, 4. Let $\{t, r, \theta, \varphi\}$ be the usual spherical coordinates in Minkowski spacetime.

If we try to solve the (effective) Einstein-Hilbert equations (in the zero mass graviton case) for the field generated by a point mass at the origin of the coordinate system we get immediately that the following "metric" fields are solutions of those equations,

$$
\mathbf{g}_{s}=\left(1-\frac{2 m}{r}\right) d t \otimes d t-\left(1-\frac{2 m}{r}\right)^{-1} d r \otimes d r-r^{2}\left(d \theta \otimes d \theta+\sin ^{2} \theta d \varphi \otimes d \varphi\right),
$$

and

$$
\begin{aligned}
g_{i} & =\left(\frac{r+\lambda-m}{r+\lambda+m}\right) d t \otimes d t-\left(\frac{r+\lambda+m}{r+\lambda-m}\right) d r \otimes d r \\
- & (r+\lambda+m)^{2}\left(d \theta \otimes d \theta+\sin ^{2} \theta d \varphi \otimes d \varphi\right),
\end{aligned}
$$

with $\lambda$ an arbitrary real parameter. Now, both solutions have the same asymptotic behavior when $r \rightarrow \infty$. Which one should we use for the descriptions of physical processes? It is important to emphasize that both metrics even if expressed in the same coordinates are diffeomorphically equivalent since it is possible to perform a coordinate transformation in Eq.(154) which makes it in the new variables to have the appearance of Eq.(155). Now, take into account that the meaning of the coordinates in each one are different since we can know what the spacetime labels mean only after we fix a metric on it. Specifically this statement means that those labels are associated with physical distances and time lapses measured by ideal rods and clocks in different ways.

But Logunov thinks that $\boldsymbol{g}_{s}$ and $\boldsymbol{g}_{i}$ given in the same coordinate basis even if diffeomorphically equivalent are physically distinguished through experiments and so fixing one of them as the correct one implies in the existence of an additional theoretical criterion and such a criterion does not exists in GR. He claims that the metric $g_{i}$ when $\lambda=0$ that satisfies the condition $\stackrel{\circ}{D}_{\gamma}\left(\sqrt{-\operatorname{det} g} g^{\gamma \kappa}\right)=0$ is the only one that fits correctly all known data on solar system experiments. 
Does the method used by astronomers methods for determining the coordinates of their probes always fix those coordinates as being the spherical coordinates of Minkowski spacetime and fix the metric to be $\boldsymbol{g}_{i}$ ? It is hard to believe in that possibility...

A last comment is in order. We start our considerations by postulating that the distortion field $h$ is symmetric since it has been constructed from the symmetric tensor field $\mathbf{h}$. However, from the general theory of plastic deformations of the Lorentz vacuum presented in 2] it is quite clear that we can construct symmetric metric tensor fields associated to non symmetric $h$ extensor fields 19 . This observation shows that the quantum theory of the gravitational field must be more complex than one where the $\boldsymbol{g}$ field is supposed to arise from the existence of a symmetric graviton field. We will return to this issue in another publication.

Acknowledgement: R. da Rocha thanks CNPq 304862/2009-6 for financial support.

\section{Appendix}

Let $(M, \stackrel{\circ}{\mathbf{g}}, \stackrel{\circ}{D})$ and $(M, \boldsymbol{g}, D)$ be the two Lorentzian structures $\stackrel{20}{20}$ on the same manifold $M$ such that

$$
\stackrel{\circ}{D} \stackrel{\circ}{g}=0, D \boldsymbol{g}=0,
$$

with the nonmetricity of $D$ relative to $\stackrel{\circ}{g}$ being given by:

$$
\mathbf{Q}:=-D \stackrel{\circ}{\mathbf{g}}
$$

Let moreover the connection coefficients of $\stackrel{\circ}{D}$ and $D$ in arbitrary coordinates $\left\{x^{\mu}\right\}$ covering $U \subset M$ be:

$$
\stackrel{\circ}{D}_{\partial_{\alpha}} d x^{\rho}=-\stackrel{\circ}{\Gamma}_{\alpha \beta}^{\rho} d x^{\beta}, D_{\partial_{\alpha}} d x^{\rho}=-\Gamma_{\alpha \beta}^{\rho} d x^{\beta},
$$

and

$$
Q_{\alpha \beta \sigma}=-D_{\alpha} \stackrel{\circ}{g}_{\beta \sigma},
$$

Define the components of the strain tensor of the connection $D$ by:

$$
S_{\alpha \beta}^{\rho}=2 \Gamma_{\alpha \beta}^{\rho}-2 \stackrel{\circ}{\Gamma}_{\alpha \beta}^{\rho} \text {. }
$$

Then

$$
\begin{aligned}
Q_{\alpha \beta \sigma} & =\frac{1}{2}\left(\stackrel{\circ}{g}_{\mu \sigma} S_{\alpha \beta}^{\mu}+\stackrel{\circ}{g}_{\beta \mu} S_{\alpha \sigma}^{\mu}\right), \\
S_{\alpha \beta}^{\rho} & =\stackrel{g}{g}^{\rho \sigma}\left(Q_{\alpha \beta \sigma}+Q_{\beta \sigma \alpha}-Q_{\sigma \alpha \beta}\right) .
\end{aligned}
$$

Also,

$$
Q_{\alpha \beta \sigma}+Q_{\sigma \alpha \beta}+Q_{\beta \sigma \alpha}=S_{\alpha \beta \sigma}+S_{\sigma \alpha \beta}+S_{\beta \sigma \alpha},
$$

\footnotetext{
${ }^{19}$ In [2] it is directly derived from the variational principle and an appropriate Lagrangian the field equations for the plastic extensor field $h$.

${ }^{20}$ More general formulas relating two arbitrary general connections may be found, e.g., in $[?, 14$.
} 
where $S_{\alpha \beta \sigma}=\stackrel{\circ}{g}_{\rho \sigma} S_{\alpha \beta}^{\rho}$.

Putting

$$
K_{\alpha \beta}^{\rho}=\frac{1}{2} S_{\alpha \beta}^{\rho} .
$$

we have

$$
K_{\alpha \beta}^{\rho}=-\frac{1}{2} \stackrel{\circ}{g}^{\rho \sigma}\left(D_{\alpha} \stackrel{\circ}{g}_{\beta \sigma}+D_{\beta} \stackrel{\circ}{g}_{\sigma \alpha}-D_{\sigma} \stackrel{\circ}{g}_{\alpha \beta}\right)
$$

The relation between the curvature tensor $R_{\mu}{ }^{\rho} \alpha \beta$ associated with the connection $D$ and the Riemann curvature tensor $\stackrel{\circ}{R}_{\mu}{ }^{\rho}{ }_{\alpha \beta}$ of the Levi-Civita connection $\stackrel{\circ}{D}$ associated with the metric $\stackrel{\circ}{\mathrm{g}}$ are given by:

$$
R_{\mu}{ }^{\rho} \alpha \beta=\stackrel{\circ}{R}_{\mu}{ }^{\rho} \alpha \beta+J_{\mu}{ }_{[\alpha \beta]},
$$

where:

$$
J_{\mu}{ }^{\rho} \alpha \beta=\stackrel{\circ}{D}_{\alpha} K_{\beta \mu}^{\rho}-K_{\beta \sigma}^{\rho} K_{\alpha \mu}^{\sigma}=D_{\alpha} K_{\beta \mu}^{\rho}-K_{\alpha \sigma}^{\rho} K_{\beta \mu}^{\sigma}+K_{\alpha \beta}^{\sigma} K_{\sigma \mu}^{\rho} .
$$

Multiplying both sides of Eq. (65) by $\frac{1}{2} \theta^{\alpha} \wedge \theta^{\beta}$ we get for the curvature 2-forms of the two connections $D$ and $\stackrel{\circ}{D}$ :

$$
\mathcal{R}_{\mu}^{\rho}=\stackrel{\mathcal{R}}{\mu}_{\mu}^{\rho}+\mathfrak{J}_{\mu}^{\rho},
$$

where we have written:

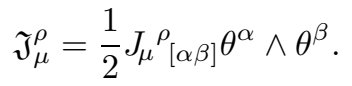

The relation between the Ricci tensor 21 of the connections $D$ and $\stackrel{\circ}{D}$ is:

$$
R_{\mu \alpha}=\stackrel{\circ}{R}_{\mu \alpha}+J_{\mu \alpha}
$$

with

$$
\begin{aligned}
J_{\mu \alpha} & =\stackrel{\circ}{D}_{\alpha} K_{\rho \mu}^{\rho}-\stackrel{\circ}{D}_{\rho} K_{\alpha \mu}^{\rho}+K_{\alpha \sigma}^{\rho} K_{\rho \mu}^{\sigma}-K_{\rho \sigma}^{\rho} K_{\alpha \mu}^{\sigma} \\
& =D_{\alpha} K_{\rho \mu}^{\rho}-D_{\rho} K_{\alpha \mu}^{\rho}-K_{\sigma \alpha}^{\rho} K_{\rho \mu}^{\sigma}+K_{\rho \sigma}^{\rho} K_{\alpha \mu}^{\sigma}
\end{aligned}
$$

Recall that the connection $\stackrel{\circ}{D}$ plays with respect to the tensor field $\boldsymbol{g}$ a role analogous to that played by the connection $D$ with respect to the metric tensor $\stackrel{\circ}{g}$ and in consequence we shall have similar equations relating these two pairs of objects. In particular, the strain of $D$ with respect to $g$ equals the negative of the strain of $D$ with respect to $\stackrel{\circ}{\mathbf{g}}$, since we have:

$$
S_{\alpha \beta}^{\rho}=\Gamma_{\alpha \beta}^{\rho}+\Gamma_{\beta \alpha}^{\rho}-\stackrel{\circ}{b}_{\alpha \beta}^{\rho}=-\left(\stackrel{\circ}{\Gamma}_{\alpha \beta}^{\rho}+\stackrel{\circ}{\Gamma}_{\beta \alpha}^{\rho}-b_{\alpha \beta}^{\rho}\right),
$$

where $b_{\alpha \beta}^{\rho}=\stackrel{\circ}{\Gamma}_{\alpha \beta}^{\rho}+\stackrel{\circ}{\Gamma}_{\beta \alpha}^{\rho}$ and $b_{\alpha \beta}^{\rho}=\Gamma_{\alpha \beta}^{\rho}+\Gamma_{\beta \alpha}^{\rho}$. Furthermore, we have that:

$$
\begin{aligned}
K_{\alpha \beta}^{\rho} & =-\frac{1}{2} \stackrel{\rho}{g}^{\rho \sigma}\left(D_{\alpha} \stackrel{\circ}{g}_{\beta \sigma}+D_{\beta} \stackrel{\circ}{g}_{\alpha \sigma}-D_{\sigma} \stackrel{\circ}{g}_{\alpha \beta}\right) \\
& =\frac{1}{2} g^{\rho \sigma}\left(\stackrel{\circ}{D}_{\alpha} g_{\beta \sigma}+\stackrel{\circ}{D}_{\beta} g_{\alpha \sigma}-\stackrel{\circ}{D}_{\sigma} g_{\alpha \beta}\right) .
\end{aligned}
$$

${ }^{21}$ For the Ricci tensor Ricci $=R_{\mu \alpha} d x^{\mu} \otimes d x^{\nu}$, we use the convention $R_{\mu \alpha}:=R_{\mu} \rho_{\alpha \rho}$. 
Now, recall that given arbitrary coordinates $\left\{x^{\alpha}\right\}$ covering $U \subset M$ and $\left\{x^{\prime \alpha}\right\}$ covering covering $V \subset M(U \cap V \neq \varnothing)$ a relative tensor $\mathfrak{A}$ of type $(r, s)$ and weight $22 w$ is a section of the bundle $23 T_{q}^{p} M \otimes\left(\bigwedge^{4} T^{*} M\right)^{\otimes w}$. We have on an arbitrary coordinate basis that

We have

$$
\mathfrak{A}=\mathfrak{A}_{\nu_{1} \ldots \nu_{s}}^{\mu_{1} \ldots \mu_{r}}\left(x^{\alpha}\right) \partial_{\mu_{1}} \otimes \cdots \otimes \partial_{\mu_{r}} \otimes d x^{\nu_{1}} \otimes \cdots \otimes d x^{\nu_{s}} \otimes(\tau)^{\otimes w}
$$

with $\tau:=d x^{0} \wedge \cdots \wedge d x^{3}$. The set of functions $\mathfrak{A}_{\nu_{1} \ldots \nu_{s}}^{\mu_{1} \ldots \mu_{r}}\left(x^{\alpha}\right)=(\sqrt{-\operatorname{det} g})^{w} A_{\nu_{1} \ldots \nu_{s}}^{\mu_{1} \ldots \mu_{r}}\left(x^{\alpha}\right)$ is said to be the components of the relative tensor field $\mathfrak{A} \in \sec \left(T_{s}^{r} M \otimes\left(\Lambda^{4} T^{*} M\right)^{w}\right)$ and under a coordinate transformation $x^{\alpha} \mapsto x^{\prime \beta}$ with Jacobian $J=\operatorname{det}\left(\frac{\partial x^{\prime \alpha}}{\partial x^{\beta}}\right)$ these functions transform as [5, 15]

$$
\mathfrak{A}_{\kappa_{1} \ldots \kappa_{s}}^{\prime \lambda_{1} \ldots \lambda_{r}}\left(x^{\prime \beta}\right)=J^{w} \frac{\partial x^{\prime \lambda_{1}}}{\partial x^{\mu_{1}}} \ldots \frac{\partial x^{\prime \lambda_{1}}}{\partial x^{\mu_{1}}} \frac{\partial x^{\nu_{1}}}{\partial x^{\kappa_{1}}} \ldots \frac{\partial x^{\nu_{s}}}{\partial x^{\kappa_{s}}} \mathfrak{A}_{\nu_{1} \ldots \nu_{s}}^{\mu_{1} \ldots \mu_{r}}\left(x^{\alpha}\right) .
$$

On a manifold $M$ equipped with a metric tensor field $\boldsymbol{g}$ we can write $\mathfrak{A}_{\nu_{1} \ldots \nu_{s}}^{\mu_{1} \ldots \mu_{r}}\left(x^{\alpha}\right)=$ $(\sqrt{-\operatorname{det} g})^{w} A_{\nu_{1} \ldots \nu_{s}}^{\mu_{1} \ldots \mu_{r}}\left(x^{\alpha}\right)$ where the $A_{\nu_{1} \ldots \nu_{s}}^{\mu_{1} \ldots \mu_{r}}\left(x^{\alpha}\right)$ are the components of a tensor field $A \in \sec T_{s}^{r} M$.

The covariant derivative of a relative tensor field relative to a given arbitrary connection $\nabla$ defined on $M$ such that $\nabla_{\frac{\partial}{x^{\nu}}} d x^{\mu}=-\mathbf{L}_{\nu \alpha}^{\mu} d x^{\alpha}$ is given (as the reader may easily find) by

$$
\nabla_{\partial_{\kappa}} \mathfrak{A}:=\left(\nabla_{\kappa} \mathfrak{A}_{\nu_{1} \ldots \nu_{s}}^{\mu_{1} \ldots \mu_{r}}\right) \partial_{\mu_{1}} \otimes \cdots \otimes \partial_{\mu_{r}} \otimes d x^{\nu_{1}} \otimes \cdots \otimes d x^{\nu_{s}} \otimes(\tau)^{\otimes w}
$$

where

$$
\begin{aligned}
\nabla_{\kappa} \mathfrak{A}_{\nu_{1} \ldots \nu_{s}}^{\mu_{1} \ldots \mu_{r}} & =\frac{\partial}{\partial x^{\kappa}} \mathfrak{A}_{\nu_{1} \ldots \nu_{s}}^{\mu_{1} \ldots \mu_{r}}+\mathbf{L}_{\iota \kappa}^{\mu_{p}} \mathfrak{A}_{\nu_{1} \ldots \mu_{p-1} \iota \mu_{p+1} \ldots \mu_{r}}^{\mu_{1} \ldots \ldots \ldots \ldots \nu_{s}} \\
& -\mathbf{L}_{\nu_{q} \kappa}^{\iota} \mathfrak{A}_{\nu_{1} \ldots \nu_{q-1} \iota \nu_{q+1} \ldots \nu_{s}}^{\mu_{1} \ldots \ldots \ldots \ldots \ldots \mu_{r}}-w \mathbf{L}_{\kappa \sigma}^{\sigma} \mathfrak{A}_{\nu_{1} \ldots \nu_{s}}^{\mu_{1} \ldots \mu_{r}}
\end{aligned}
$$

In particular for the Levi-Civita connection $\stackrel{\circ}{D}$ of $\stackrel{\circ}{g}$ and $D$ of $\boldsymbol{g}$ we have for the relative tensors $\sqrt{-\operatorname{det} \stackrel{\circ}{g}} \otimes d x^{0} \wedge \cdots \wedge d x^{3}$ and $\sqrt{-\operatorname{det} \boldsymbol{g}} \otimes d x^{0} \wedge \cdots \wedge d x^{3}$ that:

$$
\begin{aligned}
\stackrel{\circ}{D}_{\alpha}(\sqrt{-\operatorname{det} \stackrel{\circ}{\boldsymbol{g}}}) & =\partial_{\gamma}(-\operatorname{det} \stackrel{\circ}{\boldsymbol{g}})-\stackrel{\circ}{\Gamma}_{\gamma \rho}^{\rho}-\operatorname{det} \stackrel{\circ}{\boldsymbol{g}}=0, \\
\stackrel{\circ}{D}_{\alpha}\left(\frac{1}{\sqrt{-\operatorname{det} \stackrel{\circ}{g}}}\right) & =\partial_{\gamma}\left(\frac{1}{\sqrt{-\operatorname{det} \stackrel{\circ}{g}}}\right)+\stackrel{\circ}{\Gamma}_{\gamma \rho}^{\rho} \frac{1}{-\operatorname{det} \stackrel{\circ}{g}}=0, \\
D_{\alpha}(\sqrt{-\operatorname{det} \boldsymbol{g}}) & =\partial_{\gamma}(\sqrt{\operatorname{det} \boldsymbol{g}})-\Gamma_{\gamma \rho}^{\rho} \sqrt{\operatorname{det} \boldsymbol{g}}=0, \\
D_{\alpha}\left(\frac{1}{\sqrt{-\operatorname{det} \boldsymbol{g}}}\right) & =\partial_{\gamma}\left(\frac{1}{\sqrt{-\operatorname{det} \boldsymbol{g}}}\right)+\Gamma_{\gamma \rho}^{\rho} \frac{1}{\sqrt{-\operatorname{det} \boldsymbol{g}}}=0 .
\end{aligned}
$$

\footnotetext{
${ }^{22}$ The number $w$ is an integer. Of course, if $w=0$ we are back to tensor fields.

${ }^{23}$ The notation $\left(\bigwedge^{4} T^{*} M\right)^{\otimes w}$ means the $w$-fold tensor product of $\bigwedge^{4} T^{*} M$ with itself.
} 
Now, if we define

$$
\varkappa:=\sqrt{\frac{\operatorname{det} g}{\operatorname{det} g}},
$$

we can easily prove the the following relations:

$$
\begin{aligned}
& K_{\rho \sigma}^{\rho}=-\frac{1}{2} \stackrel{\circ}{g}^{\alpha \beta} D_{\sigma} \stackrel{\circ}{\alpha \beta}_{\alpha \beta}=\frac{1}{2} g^{\alpha \beta} \stackrel{\circ}{D}_{\sigma} g_{\alpha \beta}=\frac{1}{\varkappa} \partial_{\sigma}(\varkappa) \\
& g^{\alpha \beta} K_{\alpha \beta}^{\rho}=-\frac{1}{\varkappa} \stackrel{\circ}{D}_{\sigma}\left(\varkappa g^{\rho \sigma}\right)=-\frac{1}{\sqrt{-\operatorname{det} \boldsymbol{g}}} \stackrel{\circ}{D}_{\sigma}\left(\sqrt{-\operatorname{det} \boldsymbol{g}} g^{\rho \sigma}\right) \\
& \stackrel{\circ}{g}^{\alpha \beta} K_{\alpha \beta}^{\rho}=\frac{1}{\varkappa^{-1}} D_{\sigma}\left(\varkappa^{-1} \stackrel{g}{g}^{\rho \sigma}\right) .
\end{aligned}
$$

Another useful formulas valid for our particular connections $\stackrel{\circ}{D}$ and $D$ are:

$$
\begin{aligned}
& \stackrel{\circ}{D}_{\alpha} K_{\rho \beta}^{\rho}=\stackrel{\circ}{D}_{\beta} K_{\rho \alpha}^{\rho} \\
& D_{\alpha} K_{\rho \beta}^{\rho}=D_{\beta} K_{\rho \alpha}^{\rho} .
\end{aligned}
$$

\section{References}

[1] Barnes, K. J., Lagrangian Theory for the Second-Rank Tensor Field, J. Math. Phys. 6, 788-794 (1965).

[2] Fernández, V.V., and Rodrigues, W. A. Jr., Gravitation as a Plastic Distortion of the Lorentz Vacuum, Fundamental Theories of Physics 168, Springer, Heidelberg, 2010.

[3] Logunov, A. A., Mestvirishvili, M., The Relativistic Theory of Gravitation, Mir Publ., Moscow, 1989.

[4] Logunov, A. A., Relativistic Theory of Gravity, Nova Science Publ., New York, 1999.

[5] Lovelok, D., and Rund, H., Tensors, Differential Forms, and Variational Principles, J. Wiley \& Sons, New York, 1975.

[6] Rodrigues, W. A. Jr., and Oliveira, E. Capelas, The Many Faces of Maxwell, Dirac and Einstein Equations. A Clifford Bundle Approach. Lecture Notes in Physics 722, Springer, Heidelberg, 2007.

[7] Notte-Cuello, E. A., and Rodrigues, W. A. Jr., A Maxwell Like Formulation of the Gravitational Theory in Minkowski Spacetime, Int. J. Mod. Phys. D 16, 1027-1041 (2007). [arXiv:gr-qc/0612098v3]

[8] Notte-Cuello, E. A., and Rodrigues, W. A. Jr., Freud's Identity of Differential Geometry, the Einstein-Hilbert Equations and the Vexatious Problem of the Energy-Momentum Conservation in GR, Adv. Appl. Clifford Algebras 19, 113-145 (2009). [arXiv:0801 .2559v4 [math-phys]] 
[9] Notte-Cuello, E., Rodrigues, W. A. Jr., and Souza, Q. A. G., The Square of the Dirac and spin-Dirac Operators on a Riemann-Cartan Space(time), Rep. Math. Phys. 60, 135-157 (2007).

[10] Frankel T., The Geometry of Physics, Cambridge University Press, Cambridge, 1997.

[11] Fronsdal, C., Massless Fields with Integer Spin, Phys. Rev. D 18, 3624-3629 (1978).

[12] Møller, C., Further Remarks on the Localization of the Energy in the General Theory of Relativity,Ann. Phys. 12, 118-133 (1961).

[13] Padmanabhan T., From Gravitons to Gravity: Myths and Reality, Int. J. Mod. Phys. D 17, 367-398 (2008). [arXiv:gr-qc/0409089v1]

[14] Souza, Q. A. G. and Rodrigues, W. A. Jr., The Dirac Operator and the Structure of Riemann-Cartan-Weyl Spaces, in Letelier, P. A. , and Rodrigues. W. A. Jr. (eds.), Gravitation: The Spacetime Structure, Proc. of the 8th Latin American Symposium on Relativity and Gravitation, Aguas de Lindóia, July 1993, World Sci., Singapore (1994).

[15] Tiee, C., Contravariance, Covariance, Densities, and all That: An Informal Discussion on Tensor Calculus, 2006. http://math.ucsd.edu/ ${ }^{\sim}$ ctiee/tensors.pdf

[16] Thirring, W., A Course in Mathematical Physics, vol.2, Springer-Verlag, New York, 1979.

[17] Zorawski, M., Theorie Mathematiques des Dislocations, Dunod, Paris, 1967. 